\title{
Validation of different soft tissue simulation methods for breast augmentation
}

\author{
Liesbet Roose ${ }^{\mathrm{a}, *, 1}$, Wim De Maerteleire ${ }^{\mathrm{b}}$, Wouter Mollemans ${ }^{\mathrm{a}}$, \\ Paul Suetens ${ }^{\mathrm{a}}$
}

\author{
a Medical Image Computing (Radiology and ESAT/PSI), Faculties of Medicine and Engineering, \\ University Hospital Gasthuisberg, Herestraat 49, Leuven B-3000, Belgium \\ b $3 D$ Medical BV, Keizer Karel V Singel 14, Eindhoven 5615 PE, The Netherlands
}

\begin{abstract}
In spite of the large number of breast reconstruction and breast augmentation procedures, there exists no reliable simulation method to predict the patient-specific surgical outcome of breast augmentation. In this paper, we present a computational model capable of simulating the postoperative shape of the breast up to $1 \mathrm{~cm}$ accuracy after a subglandular breast implantation. In particular, we compare a mass spring system with a finite element model, we investigate the influence of different elasticity models, and we examine which imaging modalities are suited to generate the patient specific data. (C) 2005 CARS \& Elsevier B.V. All rights reserved.
\end{abstract}

Keywords: Soft tissue simulation; Augmentation mammoplasty; Mass spring system; Surgery planning

\section{Introduction}

Breast cancer is one of the most widespread diseases in the Western world: one out of eight woman will develop breast cancer before the age of 75 years. An operative mastectomy in which the breast is amputated will often be part of the treatment. In this situation, women often choose a reconstruction of the breast with a breast implant. Besides this reconstruction surgery, there is also a growing request for breast augmentation because of aesthetic improvement (augmentation mammoplasty). Due to the large number of augmentation procedures, the variety of available implant sizes and shapes is growing

\footnotetext{
* Corresponding author.

E-mail address: liesbet.roose@esat.kuleuven.ac.be (L. Roose).

${ }^{1}$ Liesbet Roose is Research Assistant of the Fund for Scientific Research-Flanders (Belgium) (FWOVlaanderen).
} 
vastly. Therefore preoperative implant selection has become a major issue to ensure a natural and symmetrical appearance.

Our goal is the development of a computer-based planning tool to predict the surgical outcome of augmenting procedures, using a patient-specific representation based on medical images. In this paper, we will focus on subglandular placement only, where the implant is placed above the pectoralis major muscle. Williams et al. [1] proposed a method for the simulation of breast augmentation, but her research still lacks validation. In search for the simplest computational method that offers a sufficiently accurate approximation of the postoperative shape of the breast, we investigate and validate different aspects of the problem solving strategy.

First, we examine different imaging modalities that can be used to build the geometry of the representation. Secondly, we work out a framework to model the behaviour of the soft tissue using a mass spring system (MSS), and compare this with the finite element model (FEM). Finally, we investigate the importance of an accurate estimation of the elastic tissue characteristics and compare two approaches: considering the breast as a homogeneous tissue versus an approach using multiple tissue types.

In this first modeling attempt, we do not take biological effects into account, even though we know that tissue growth and atrophy can have a large influence. We strive for a realistically looking result, and we want to simulate the postoperative shape with a maximal error of around $1 \mathrm{~cm}$, which is clinically acceptable.

\section{Geometric modeling}

Different imaging modalities can be used to extract the 3D geometry of the breast, from which MR or CT scans are the easiest to build a mesh upon. However, they require an expensive and time-consuming scan from every patient. If we do not need segmented data, another possibility is to use a 3D camera to capture the geometry of the skin surface and to combine this with information about the thickness of the fatty tissue layer to build a patientspecific volumetric representation. To estimate the tissue thickness, palpation or ultrasound measurements can be used. The advantage of this technique is that it is a relatively cheap and fast method, and the plastic surgeon himself can easily provide all necessary data.

From the volumetric model of the tissue, a 3D mesh has to be built, for which tetrahedral and layer-based hexahedral meshes are the most common topologies. We have chosen tetrahedral meshes because of their flexibility in representing non-layered geometries [2]. We use the Netgen [3] software to build the volumetric mesh.

\section{Computational method: MSS versus FEM}

FEM is based on continuum mechanics and is widely accepted as an accurate method to model deformations, but is expensive in computational cost and memory usage. Most approaches for breast modeling are FEM-based (e.g., Refs. [4,5]). These models are, however, specifically designed for applications which require higher accuracy than the application described here. The less accurate mass spring models are an attractive alternative to FEM because of their conceptual simplicity, easy implementation, and short computation time. These models have been used in various applications, first in computer graphics, later also in anatomical modeling. Examples of the latter are real-time simulation 

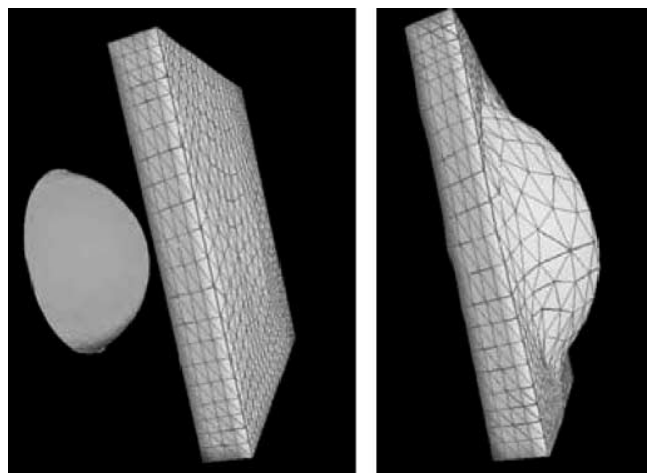

Fig. 1. On the left, the undeformed plate of tissue and the implant. On the right, the tissue after the implant had been placed.

of a muscle [6], a stomach model for the analysis of X-ray images of this organ [7], and prediction of the surgical outcome of facial tissue in a craniofacial operation $[8,9]$.

In the classical mass spring model [9], the tissue is discretized in $n$ points at position $p_{i}$ for $i=1 \ldots n$, each with mass $m_{i}$. These points are connected by elastic springs and dampers (with damping factor $g$ ), and their movement is described by the following differential equation:

$$
m_{i} \frac{\mathrm{d}^{2} p_{i}(t)}{\mathrm{d} t^{2}}+\gamma \frac{\mathrm{d} p_{i}(t)}{\mathrm{d} t}+F_{i}^{\mathrm{int}}(t)=-F_{i}^{\text {ext }}(t)
$$

where $F_{i}^{\text {int }}$ denotes the resulting internal elastic force caused by strains of the adjacent springs and $F_{i}^{\text {ext }}$ is the sum of external forces (gravity for instance).

To compute an equilibrium situation after the application of forces or deformations, we use the approach proposed by Teschner [8]. Instead of solving for the differential equation (Eq. (1)), the estimate of the deformation is formulated as an unconstrained minimization problem. Traditionally, these problems are solved by the steepest descent, conjugate gradient, trust region methods, or Newton-based methods [10], which require the computation of the gradient (and in the latter case of the Hessian). However, we use another approach, based on physical intuition, expecting the mean force to decline if we move the points in the direction of the force acting on it, as has been proposed in Ref. [9].

To compare FEM and MSS, we simulate the placement of a sphere segment under a rectangular plate of tissue, as shown in Fig. 1. Using a plate of size $30 \times 250 \times 250 \mathrm{~mm}$ and different implants with a height varying from $29 \mathrm{~mm}$ to $46 \mathrm{~mm}$, we compute the tissue evolution with the MSS implementation described above and with a conventional FEM method. The maximal error between corresponding points in the two simulations varies from 3.8 to $8.9 \mathrm{~mm}$, depending on the height of the implant. Keeping in mind that we strive for 1 $\mathrm{cm}$ accuracy, we can conclude that a mass spring representation suffices for this application.

\section{Occurrence of non-physical minima in MSS}

Because of the large tissue deformation in this application, the simulation of the surgical procedure is done in two phases. In a first phase, the mass points right above the pectoralis major are moved forward to create space for the insertion of the implant. This is done by moving these points in small steps, while the positions of the other points are 
(a)

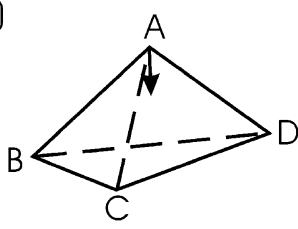

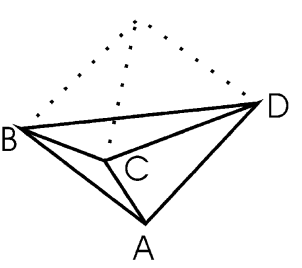

(b)

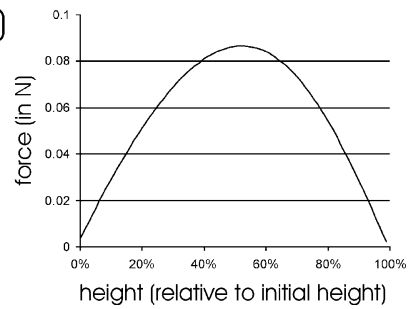

Fig. 2. (a) Both the left and the right tetrahedra are in equilibrium; however, the right one is not a possible physical deformation from the left tetrahedron. (b) The evolution of the force component in the direction perpendicular to the ground plane on point $\mathrm{A}$ if this point moves down.

relaxed, but exact computations of the intermediate configurations are not necessary. After this first phase, the implant can be placed and, in the second phase, an accurate calculation of the final equilibrium is performed.

In both phases, it is important to be aware of non-physical local minima of the mass spring system, which can occur because of the discretization of mass and forces in this approach (see Figs. 2a and 3).

The risk of a flip-over of a tetrahedron increases rapidly if a tetrahedron becomes flattened, which is not unusual in case of large deformations. We illustrate this with Fig. 2: if point $\mathrm{A}$ is moving down to the ground plane, the forces to counteract this movement are aligned along the springs $\mathrm{AB}, \mathrm{AC}$, and $\mathrm{AD}$. However, these springs become more parallel to the ground plane if point A moves downwards and the transversal component of the forces on these springs becomes smaller. Fig. 2(b) shows the evolution of the component of the force perpendicular to the ground plane. It can clearly be seen that the resistance to this movement declines if the tetrahedron becomes flattened.

The flip-over of tetrahedron happens mostly in the first phase, in which the largest deformation of the mass spring model takes place. In the second phase, a flip-over of tetrahedra will only occur at the sites where skin has a high curvature, for instance just above the borders of the implant.

To prevent this undesired behaviour, we add an additional check to the optimization algorithm. If a tetrahedron would flip over because of the displacement of a point, we do not allow this movement. Instead, we iteratively try to move the point in the same direction, each time over a smaller distance. Only if this fails in five subsequent iterations, we leave the point in its original position. Doing so, we can use a rather large global step length in the optimization algorithm, but we avoid non-physical local minima. Disadvantage of this precautionary procedure is the doubling of computational cost.
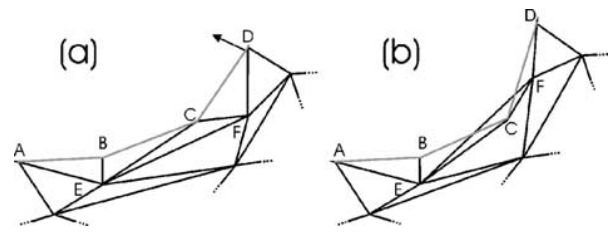

Fig. 3. Line ABCD represents the skin surface in a triangular mesh. In (b), the local curvature of the skin is increased, which causes triangle CEF to flip. The behaviour of a tetrahedral mesh in $3 \mathrm{D}$ is more difficult to visualize, but is analogous to this triangular mesh in $2 \mathrm{D}$. 


\section{Importance of tissue elasticity}

Apart from microscopic structures, the female breast consists of fatty and glandular tissues. Various estimates of the stress-strain behaviour of fatty and glandular tissues in the breast have been proposed: linear as well as non-linear (see, e.g., Ref. [5]). However, most descriptions are valid only for small displacements, whereas the deformations caused by a breast augmentation can be fairly large (typically a spring can have up to 2.5 times its original length). Ruiter [5] already pointed out that exponential elasticity models are implausible for large deformations because they result in physically unrealistic high moduli of Young. We therefore only examine the polynomial model from Samani [4] and the linear models from Bakic [11] and Lorenzen et al. [12] to investigate the effect from the different elasticity models. In a first test, we construct an inhomogeneous tissue model from a CT scan of a real patient, in which fatty and glandular tissues were distinguished. We compare the result of an augmentation simulation on this inhomogeneous model with the simulation of a similar augmentation, assuming all tissues are homogeneously fat. The results in Table 1 show that it is not necessary to use an inhomogeneous model, which makes the segmentation between the different tissue types unnecessary.

Secondly, we compare the different models for elasticity, using homogeneous tissues. In the three tested elastic models, the modulus of Young varies from $1.7 \mathrm{kPa}$ to $500 \mathrm{kPa}$, but the maximal error between the different simulations is only $5 \%$ from the total displacement and below $2.5 \mathrm{~mm}$. We can therefore conclude that the modulus of Young has little impact on the final result, and that we can use a homogeneous tissue with an arbitrarily chosen model for the tissue elasticity. This result corresponds to similar conclusions in Ref. [5].

\section{Validation}

We simulate the implantation of a 245-g silicone-filled implant for aesthetic improvement by a 30 -year-old woman with our mass spring implementation. We use a preoperative 3D picture of this patient to build the mesh, and compare our simulation with a postoperative 3D picture. The result can be seen in Fig. 4. The largest errors are found at the nipple and at the bottom and top of the breast, where the stresses are the largest. The maximum error on the breast surface is $8.5 \mathrm{~mm}$.

\section{Discussion}

The method developed in this paper is a first step towards breast augmentation simulation. We described a computational method to predict the postoperative shape of the breast after a breast augmentation, without taking biological effects such as growth into account. Our method is based on a mass spring representation of the tissue and we successfully managed to prevent non-physical minima, but the testing necessary for it decreases the speed advantage from the mass spring model. Therefore, in further research, we plan to examine also finite element models.

Table 1

Difference between a simulation on a homogeneous and an inhomogeneous tissue model

\begin{tabular}{lr}
\hline Elasticity model & Maximal difference (mm) \\
\hline Wellman_Samani & 2.21 \\
Bakic & 2.18 \\
Lorenzen & 2.05 \\
\hline
\end{tabular}


(a)

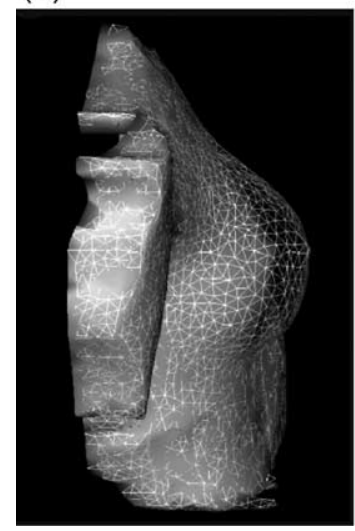

(b)

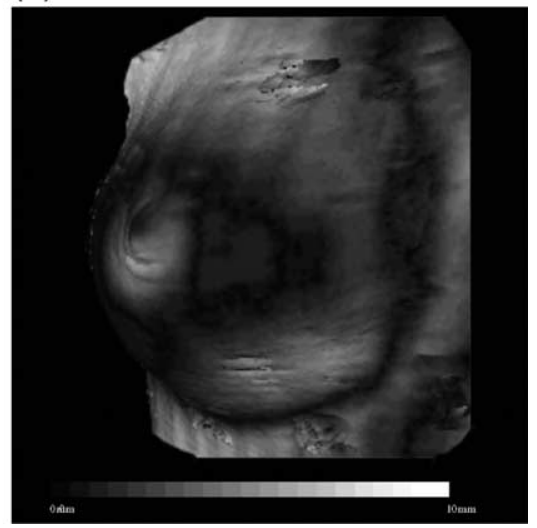

Fig. 4. (a) Combination of the preoperative breast (solid) and the postoperative shape (wireframe). (b) Visualisation of the difference between the real and the simulated postoperative breast. Dark points represent the smallest error; bright points represent the largest error.

The validation on patient data shows that the errors are larger at sites where stresses are high. We expect this to be a result of the biological effects of growth and atrophy, resulting from these stresses. This encourages us to investigate these and try to incorporate them into future extensions of our model.

\section{Acknowledgments}

This work is part of the Flemish government IWT GBOU 0201095 project.

\section{References}

[1] C. Williams, et al., Simulation studies for predicting surgical outcomes in breast reconstructive surgery, MICCAI (2003).

[2] H. Delingette, Towards realistic soft tissue modeling in medical simulation, Technical Report, vol. 3506 , INRIA, Sophia-Antipolis, 1998

[3] Netgen, http://www.hpfem.jku.at/netgen/.

[4] A. Samani, et al., Biomechanical 3-D finite element modeling of the human breast for MR/X-ray using MRI data, IEEE Transactions on Medical Imaging 20 (4) (2001).

[5] N. Ruiter, Registration of X-ray mammograms and MR-volumes of the female breast based on simulated mammographic deformation. PhD Thesis, Universität Mannheim, 2003.

[6] L.P. Nedel, D. Thalmann, Real time muscle deformations using mass-spring systems, Proceedings Computer Graphics International (1998).

[7] Y. Kita, Elastic-model driven analysis of several views of a deformable cylindrical object, IEEE Transactions on Pattern Analysis and Machine Intelligence 18 (12) (1996).

[8] M. Teschner, Direct computation of soft-tissue deformation in craniofacial surgery simulation, Berichte aus der Kommunikations-und Informationstechnik, vol. 20, Shaker Verlag, Aachen, 2001.

[9] W. Mollemans, et al., Tetrahedral mass spring model for fast soft tissue deformation, IS4TM (2003).

[10] J. Nocedal, S.J. Wright, Numerical Optimization, Springer-Verlag, 1999.

[11] P.R. Bakic, Breast tissue description and modeling in mammography, PhD Thesis, Lehigh University, 2000.

[12] J. Lorenzen, et al., MR elastography of the breast: preliminary clinical results, Fortschritte auf dem Gebiet der Röntgenstrahlen und der bildgebenden Verfahren 174 (7) (2002). 